\title{
Covalent functionalization of graphene oxide with flame retardant and its effect on thermal stability and flame retardancy of epoxy composites
}

WEIZHAO HU ${ }^{a}$, LEI SONG ${ }^{a}$, JIAN WANG ${ }^{a, b *}$ YUANG HU ${ }^{a, b * *}$ and PING ZHANG

a State Key Laboratory of Fire Science, University of Science and Technology of China, 96 Jinzhai Road, Hefei, Anhui 230026, P.R. China.

${ }^{b}$ USTC-CityU Joint Advanced Research Centre, Suzhou Key Laboratory of Urban Public Safety, Suzhou Institute for Advanced Study, University of Science and Technology of China, 166 Ren'ai Road Suzhou, Jiangsu 215123, P.R. China.

c Southwest University of Science and Technology, 59 Qinglong Road, Mianyang, Sichuan 621010, P.R. China.

\begin{abstract}
In order to improve the dispersion and fire retardant property in epoxy resin (EP), graphene oxide (GO) was functionalized via surface modification by a flame retardant which was synthesized by the reaction of methyl dichlorophosphate and 10-(2,5-dihydroxyl-phenyl)-9,10-dihydro-9-oxa-10-phosphaphenan-threne-10-oxide (DOPO-BQ). The property of functionalized GO (FGO) was characterized by fourier transform infrared spectroscopy, $\mathrm{H}$ - and $\mathrm{P}$ - nuclear magnetic resonance, $\mathrm{X}$-Ray photoelectron spectroscopy and thermogravimetric analysis (TGA). Series different ratios of GO- and FGO- epoxy nanocomposites were obtained by in situ polymerization. The incorporation of FGO enhanced the thermal stability and flame retardancy of epoxy nanocomposites effectively. The thermal properties of the nanocomposites were investigated by TGA test in nitrogen atmosphere, indicating that FGO can improve the char residues. The flame retardancy of the nanocomposites was characterized by cone calorimeter test. The results showed that the incorporation of $2 \mathrm{wt} \%$ FGO into EP decreased the value of peak heat release rate, total heat release, average effective heat of combustion, peak values of the $\mathrm{CO}$ release rate and $\mathrm{CO}_{2}$ release rate by $25 \%, 28 \%$, $29.5 \%, 27 \%$ and $29 \%$, respectively. This work confirms that the FGO is an effective solution for improving the thermal stability and flame retardancy of epoxy resins.
\end{abstract}

KEYWORDS: graphene oxide; functionalization; flame retardant; epoxy.

\section{INTRODUCTION}

Graphene, as a new emerging carbon-based material, has attracted increasing attention from industrial to academic fields owing to its thermal, mechanical, and electrical properties. [1-3] With these excellent properties, significant enhancements on the thermal, mechanical, electrical and gas barrier properties are achieved by introducing very low loadings of graphene into polymer matrix. [4-7] Like other layered materials, the unique single-atom two-dimensional sheet structure, composing of $\mathrm{sp} 2$ carbon atoms in a hexagonal lattice, has made graphene as a promising flame retardant additive for reducing the fire hazards of the polymer materials. To date, graphene oxide or its derivatives has been incorporated into various kinds of polymers such as Poly (vinyl alcohol) [8], epoxy resins [9-10], polystyrene [11], and ethylene-vinyl acetate [12], investigating the peak heat release rate and smoke release of nanocomposites. However, the irreversible aggregation or restacking of graphene in polymer materials limits their industrial application due to the intrinsic van der Waals force between graphene layers interferes. [13-15] Then the chemical modification could improve the dispersion of graphene in polymer matrices, but general organic compounds may not have effective efficiency to improve the flame retardancy of nanocomposites. Thus, the using of flame retardants to treat the surface of graphene with simultaneously improved dispersion and fire resistance is a feasible approach.

Over the past few years, the halogen-free products such as phosphorus, silicon, and nitrogen compounds have been developed in recent years. [16-18] Phosphorus-containing compounds, one of the promising

\footnotetext{
${ }^{*}$ Corresponding Author. Tel./fax: +86-551-63606463.

E-mail:wangj@ustc.edu.cn

${ }^{* *}$ Corresponding Author. Tel./fax: +86-551-63601664.

E-mail: yuanhu@ustc.edu.cn
} 
halogen-free FRs, are widely applied to various polymer materials due to its high flame retardant efficiency and non-toxic.[19] Among them, 9,10-dihydro-9-oxa-10- phosphaphenanthrene 10-oxide (DOPO), a cyclic phosphate with a diphenyl structure, has attracted considerable attention due to its high thermal stability, good oxidation and water resistance. It's reported that the P-H bond of DOPO could react with epoxide group of epoxy resins or other groups, indicating DOPO or its derivatives can be used as either reactive or nonreactive FRs for epoxy resins. $[20,21]$ In the open literature, both the DOPO and its derivatives exhibited high flame retardancy and thermal stability effect on epoxy resins (EP). [22-24] As for the flame retardant mechanism, DOPO mainly play its role in the gas phase via flame inhibition, or in the condensed phase as char formation catalyst at the same time.

Epoxy resins, which have been widely used in surface coating, adhesives, painting and potting materials, laminates, printed circuit boards and insulating materials for electric devices, etc, owing to their excellent mechanical and chemical properties, are very important thermosetting material. [25-28] However, the easy flammability of EP limits its wide range of applications, especially in electronic fields where high flame retardancy is required. To improve the fire resistance efficiency of EP, the combination of graphene with DOPO derivative flame retardant synergistic system is developed. The DOPO-containing polymeric flame retardant is synthesized via the reaction of methyl dichlorophosphate and 10-(2,5-dihydroxyl-phenyl)-9,10-dihydro-9-oxa-10-phosphaphenan-threne- 10-oxide (DOPO-BQ), and subsequently grafted on the surface of graphene oxide. Then the FGO were incorporated into EP to fabricate FGO/EP nanocomposites with different FGO loadings. The structure and morphology of functionalized graphene oxide was characterized by fourier transform infrared spectroscopy (FTIR), nuclear magnetic resonance (NMR), X-Ray photoelectron spectroscopy (XPS) and thermogravimetric analysis (TGA). The thermal and combustion properties of FGO/EP nanocomposites were investigated by TGA and cone calorimeter tests.

\section{EXPERIMENT}

\section{Materials}

Expandable graphite (60 mesh) was supplied by Qingdao Tianhe Graphite Co., Ltd. Potassium permanganate $\left(\mathrm{KMnO}_{4}\right)$, ammonia (25-28\% aq.), hydrazine hydrate (85\% aq.), hydrogen peroxide $\left(\mathrm{H}_{2} \mathrm{O}_{2}, 30 \%\right.$ aq.), hydrochloric acid $(\mathrm{HCl})$, sulphuric acid $\left(\mathrm{H}_{2} \mathrm{SO}_{4}, 98 \%\right)$, triethylamine, dichloromethane, methanol, toluene and tetrahydrofuran (THF) were purchased from Sinopharm Chemical Reagent Co., Ltd. Hydroxyethyl acrylate (HEA), p-benzoquinone (BQ) was purchased from Aladdin Co., Ltd. 9,10-Dihydro-9-oxa-10-phosphaphenanthrene-10-oxide (DOPO) was supplied by ShandongMingshan Fine Chemical Industry Co. Ltd. Methyldichlorophosphate (MDCP) was obtained from our group. Epoxy acrylates, which was supplied by Tianjin Tianjiao $\mathrm{Co}$, is a bisphenol A epoxy acrylate with the unsaturation concentration of $3.73 \mathrm{mmol} / \mathrm{g}$.

\section{Preparation of FGO with flame retardant}

GO was prepared by the Hummers' method and washed with THF to remove water. [29] DOPO-BQ was synthesized by the traditional method. [30]

$0.1 \mathrm{~mol}$ of DOPO-BQ and triethylamine as the acid-binding agent was dissolved in dried $200 \mathrm{ml}$ of THF into a three-neck flask equipped with a nitrogen inlet, a mechanical stirrer, refluxed and slowly heated to $60{ }^{\circ} \mathrm{C}$. $0.11 \mathrm{~g}$ of MDCP was dispersed in $40 \mathrm{ml}$ THF and then dripped into solution of flask sustained $1 \mathrm{~h}$. The flame retardant oligomer PDBMP was synthesized. The reaction mixture was stirred at $60{ }^{\circ} \mathrm{C}$ for $12 \mathrm{~h}$ under a nitrogen gas atmosphere. Then, $1 \mathrm{~g}$ of GO was added in the three-necked flask sonicated for $30 \mathrm{~min}$ and the stirred of reaction mixture was continue at $60^{\circ} \mathrm{C}$ for $12 \mathrm{~h}$ under a nitrogen gas atmosphere. After cooling, the suspension was filtered and washed with DMF and methanol, and then dried under vacuum at $50{ }^{\circ} \mathrm{C}$ for $12 \mathrm{~h}$. The FGO was prepared. The schematic process of the reaction is presented in Scheme 1. 


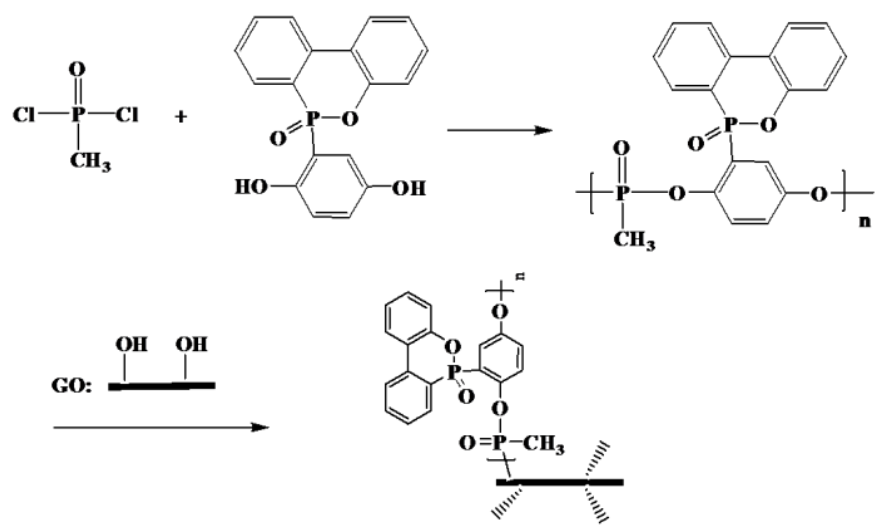

Scheme 1. Synthesis route of FGO

\section{Preparation of EP-GO and EP-FGO nanocomposites via in situ polymerization}

Typically, the required portion of GO or FGO was dispersed in THF with sonication and then added into the epoxy with $0.5 \mathrm{~h}$ of mechanical stirring and then put in a vacuum oven at $50^{\circ} \mathrm{C}$ overnight to remove the solvent. The DDM (molar ratio of epoxy group: DDM $1 / 4=4: 1$ ) was melted at $120^{\circ} \mathrm{C}$ and then added into the blending. After that the mixture was stirred for $10 \mathrm{~min}$ and poured into mold, pre-cured be prepared at $120^{\circ} \mathrm{C}$ for $2 \mathrm{~h}$ and post-cured at $150{ }^{\circ} \mathrm{C}$ for $2 \mathrm{~h}$ and finally the EP-GO and EP-FGO nanocomposites were prepared. Similarly, the different ratios of GO or FGO and EP (such as 1wt\% (EP-GO1 or EP-FGO1) and $2 \mathrm{wt} \%$ (EP-GO2 or EP-FGO2)) nanocomposites were obtained.

\section{Measurement}

FTIR spectroscopy (Nicolet 6700 FT-IR spectrophotometer) was employed to characterize FGO using thin $\mathrm{KBr}$ disc. The transition mode of the FTIR was used and the wavelength range was set from 4000 to $500 \mathrm{~cm}^{-1}$. ${ }^{1} \mathrm{H}$ NMR and ${ }^{31} \mathrm{P}$ NMR measurements were conducted on AVANCE 400 Bruker spectrometer at room temperature using $\mathrm{CDCl}_{3}$ as solvent. XPS was studied using a VG ESCALB MK-II electron spectrometer (V.G. Scientific. Ltd, UK). The excitation source was an Al Ka line at $1486.6 \mathrm{eV}$. TGA and TG-IR were carried out on TGA Q5000 IR thermogravimetric analyzer (TA instruments) and the heating rate is $20{ }^{\circ} \mathrm{C} / \mathrm{min}$. The fire safety properties of the FGO-EP nanocomposites were investigated on a Stanton Redcroft cone calorimeter according to ISO 5660-1 standard, with a heat flux of $35 \mathrm{~kW} / \mathrm{m}^{2}$. The dimensions of the samples were $100 \times$ $100 \times 3 \mathrm{~mm}^{3}$.

\section{RESULT AND DISCUSSION}

\section{Characterization of FGO}
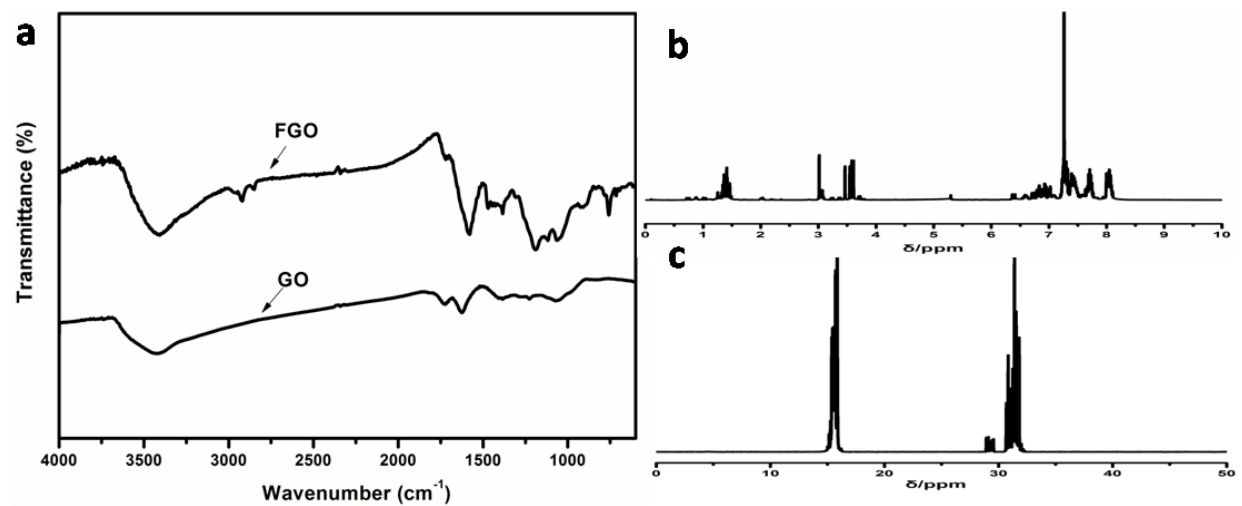

Figure 1. (a) The FTIR spectra of GO and FGO, (b) ${ }^{1} \mathrm{H}$ NMR and (c) ${ }^{31} \mathrm{P}$ NMR spectra of FGO.

The covalent linkage of flame retardant onto GO was confirmed by FTIR (Figure 1a) and ${ }^{1}$ HNMR (Figure 1b) spectra. In the spectrum of $\mathrm{GO}$, the peak at $1730 \mathrm{~cm}^{-1}$ was assigned to the $\mathrm{C}=\mathrm{O}$ stretching vibration, and the other characteristic absorption bands at 1620,1065 , and $1220 \mathrm{~cm}^{-1}$ were assigned to the stretching 
vibration of sp2 hybridized carbon, $\mathrm{C}-\mathrm{O}$ stretching, and $\mathrm{C}-\mathrm{OH}$ stretching, respectively, similar to the literature [31]. After being functionalized with PDBMP, the distinctive absorption bands appeared: the peak at $2930 \mathrm{~cm}^{-1}$ assigned to the stretching vibration of $\mathrm{CH}_{2}$; the peak at 1460 and $1590 \mathrm{~cm}^{-1}$ assigned to the $\mathrm{C}=\mathrm{C}$ group of phenyl group; the peak at 1190 and $1068 \mathrm{~cm}^{-1}$ assigned to symmetric stretching vibration and asymmetric stretching vibration of $\mathrm{P}=\mathrm{O}$ and $\mathrm{P}-\mathrm{O}$; the peak at $1311 \mathrm{~cm}^{-1}$ assigned to $\mathrm{P}-\mathrm{C}$ deformation vibration. These results clearly suggested that PDBMP had been successfully bonded to GO. As can be seen in Figure 1b, the ${ }^{1} \mathrm{H}$ NMR spectra of FGO showed that the characteristic peaks at 6.33-8.17 ppm belonged to the $\mathrm{H}$ atoms of phenyl group. And the peaks at 2.99-3.8 and 1.17-1.61 ppm were assigned to the $-\mathrm{CH}_{2}-$ and $-\mathrm{CH}_{3}$, respectively. What's more, ${ }^{31} \mathrm{P}$ NMR spectra of FGO showed that the peaks at 15.19 and $30.95 \mathrm{ppm}$ belonged to the $\mathrm{P}$ atoms linked to methyl and phenyl group respectively, and the weak peaks at $28.98 \mathrm{ppm}$ were assigned to the $\mathrm{P}$ atoms linked to phenyl group in terminal group of oligomer. All of these results indicated that PDBMP were successfully synthesized and covalently grafted onto the surface of GO.

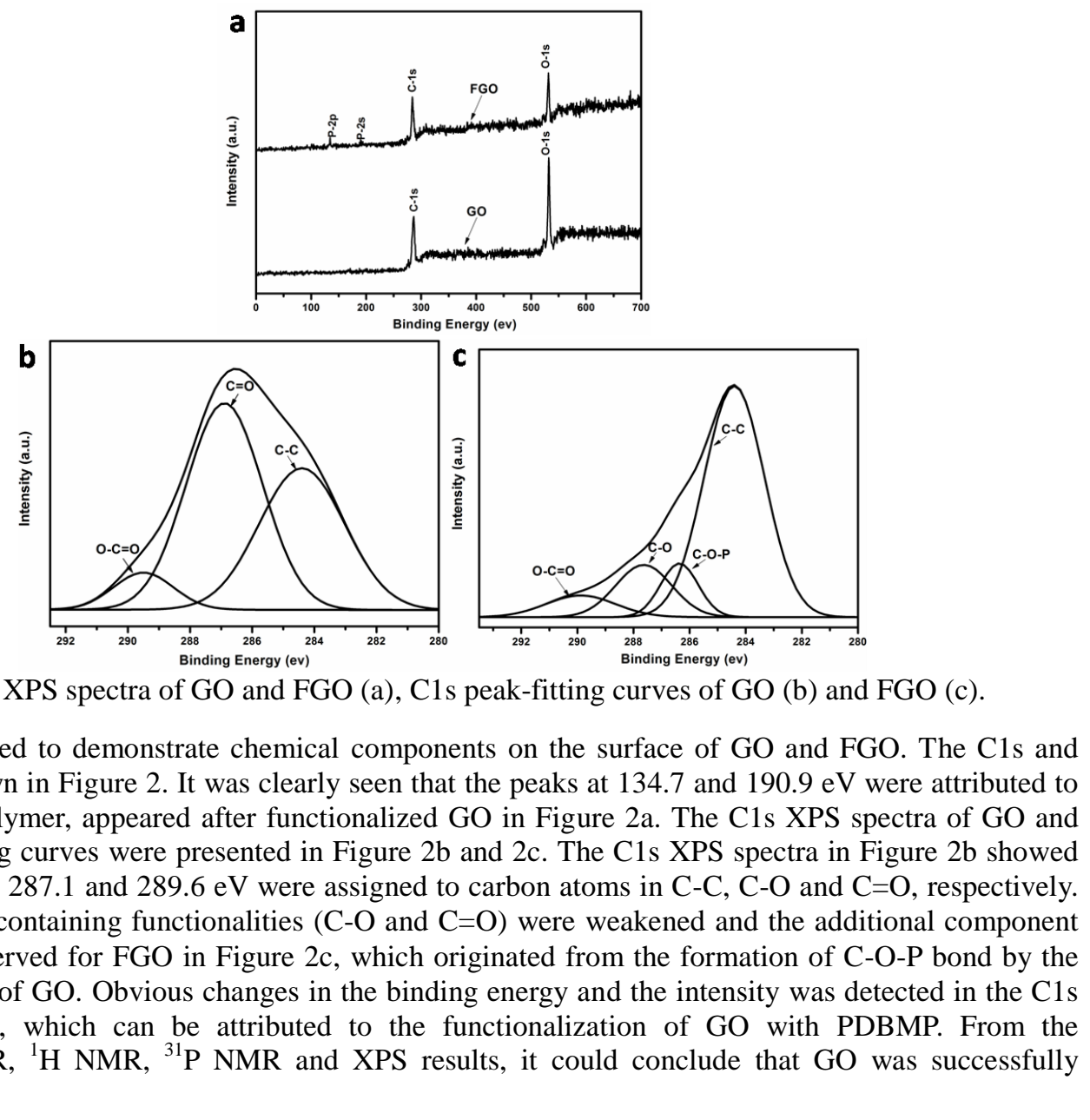

Table 1 The thermogravimetric analysis of GO and FGO in nitrogen.

\begin{tabular}{|c|c|c|c|}
\hline \multirow{2}{*}{ Sample } & \multicolumn{2}{|c|}{ Temperature at specific weight loss $\left({ }^{\circ} \mathbf{C}\right)$} & \begin{tabular}{c} 
Residues at $\mathbf{8 0 0}^{\circ} \mathbf{C}$ \\
\cline { 2 - 3 }
\end{tabular} \\
\cline { 2 - 3 } & $\mathbf{T}_{-5 \%}\left({ }^{\circ} \mathbf{C}\right)$ & $\mathbf{T}_{\max }\left({ }^{\circ} \mathbf{C}\right)$ & 53 \\
\hline GO & 112 & 193 & 67 \\
\hline FGO & 162 & 172 & 53 \\
\hline
\end{tabular}



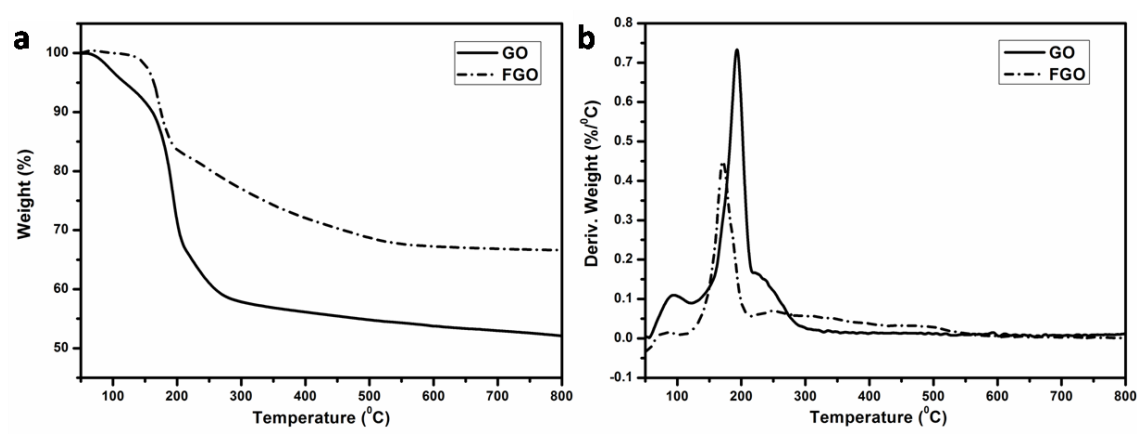

Figure 3. TGA(a) and DTG(b) curves of GO and FGO in nitrogen atmosphere.

Thermogravimetric analysis (TGA) and differential thermogravimetric (DTG) plots for the GO and FGO in nitrogen atmosphere were investigated and the results are shown in Figure 3 and Table 1. As can be seen from the curve of GO in Figure 3, the first mass loss stage below $100{ }^{\circ} \mathrm{C}$ was due to the evaporation of the adsorbed water, and the maximum mass loss temperature $\left(\mathrm{T}_{\max }\right)$ around $200{ }^{\circ} \mathrm{C}$ is caused by the decomposition of labile oxygen functional groups[32-33]. The $52.7 \%$ residual char at $800{ }^{\circ} \mathrm{C}$ was obtained after the steady loss at $300-800^{\circ} \mathrm{C}$. For FGO, the 5\% mass loss temperature $\left(\mathrm{T}_{-5 \%}\right)$ which was defined as the initial degradation temperature increased from 112 to $165^{\circ} \mathrm{C}$, suggesting that the labile oxygen groups in $\mathrm{GO}$ were partly replaced by PDBMP. The maximum weight loss of FGO around $200{ }^{\circ} \mathrm{C}$ was attributed to the residual oxygen functional groups, while the decomposition of the PDBMP in FGO was occurred between 200 and $300{ }^{\circ} \mathrm{C}$. However, the residual char of $\mathrm{FGO}$ was increased to $66.6 \%$ at $800{ }^{\circ} \mathrm{C}$, which may be due to the pyrolysis of phenyl group in PDBMP. Therefore, the TG results confirmed that the functionalization of GO not only well grafted the flame retardant onto GO but also improved the thermal stability of GO.

\section{Thermal stability of EP-GO and EP-FGO nanocomposites}

Table 2 The thermogravimetric data of EP-GO and EP-FGO nanocomposites in nitrogen.

\begin{tabular}{|c|c|c|c|}
\hline \multirow{2}{*}{ Sample } & \multicolumn{2}{|c|}{ Temperature at specific weight loss $\left({ }^{\circ} \mathbf{C}\right)$} & Residues at $\mathbf{7 0 0}^{\circ} \mathbf{C}$ \\
\cline { 2 - 3 } & $\mathbf{T}_{-\mathbf{5 \%}}\left({ }^{\circ} \mathbf{C}\right)$ & $\mathbf{T}_{\mathbf{m a x}}\left({ }^{\circ} \mathbf{C}\right)$ & 15 \\
\hline EP & 371 & 387 & 15 \\
\hline EP-GO1 & 366 & 384 & 16 \\
\hline EP-GO2 & 361 & 383 & 16 \\
\hline EP-FGO1 & 368 & 385 & 18 \\
\hline EP-FGO2 & 362 & 386 & \\
\hline
\end{tabular}
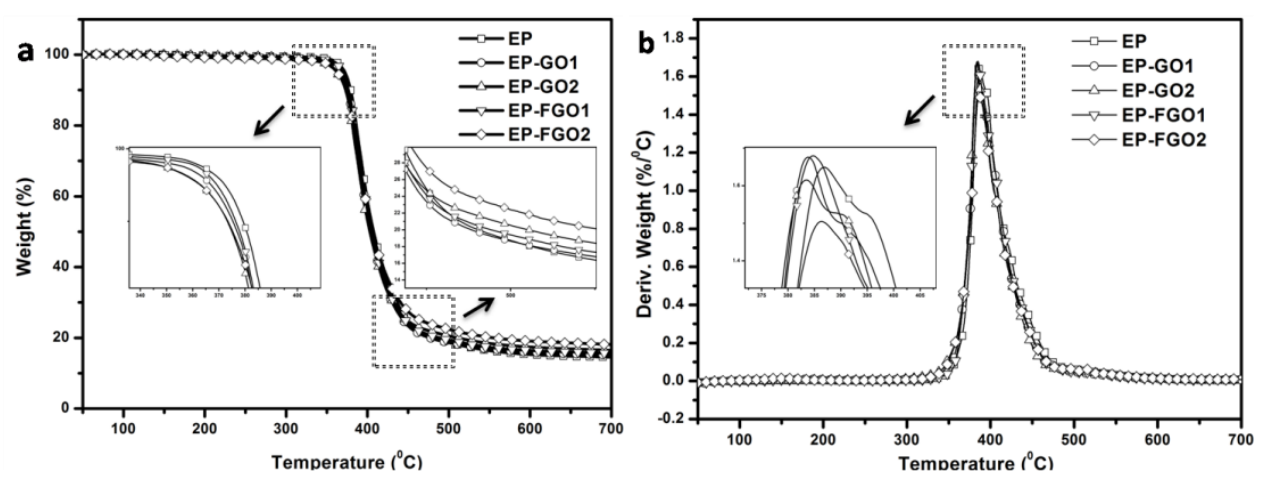

Figure 4. TGA(a) and DTG(b) curves of EP-GO and EP-FGO nanocomposites in nitrogen atmosphere.

The thermal stability and thermal degradation process of EP nanocomposites were investigated by TGA under nitrogen atmosphere. The TGA and DTG curves of EP-GO and EP-FGO nanocomposites were recorded in Figure 4 and the relative data, such as $\mathrm{T}_{-5 \%}, \mathrm{~T}_{\max }$ and the residues at $700{ }^{\circ} \mathrm{C}$, were listed in Table 2. As for the thermal degradation of neat EP, the material began to lose mass at about $360{ }^{\circ} \mathrm{C}$ and degraded almost completely before $600^{\circ} \mathrm{C}$. Only one DTG peak at about $370{ }^{\circ} \mathrm{C}$ was observed which corresponded to that the 
macromolecular chains of EP degraded and small molecular degradation products released and then formed char residue. In comparison with EP, the $\mathrm{T}_{-5 \%}$ and $\mathrm{T}_{\max }$ of EP-GO and EP-FGO nanocomposites were decreased due to the thermal degradation of GO and FGO, but the final char residues at $700{ }^{\circ} \mathrm{C}$ were increased. When the loading amount of GO increased from $1 \%$ to $2 \%$, the $\mathrm{T}_{-5 \%}$ and $\mathrm{T}_{\max }$ of EP nanocomposites decreased, which was attribute to the increase of labile oxygen functional groups on the surface of GO. However, the $\mathrm{T}_{\max }$ value was increased after the addition of FGO, which may be due to the flame retardant grafted on GO promoted the char formation. Therefore, the increase of char residue can form a barrier to protect the nanocomposites from further oxidative degradation.
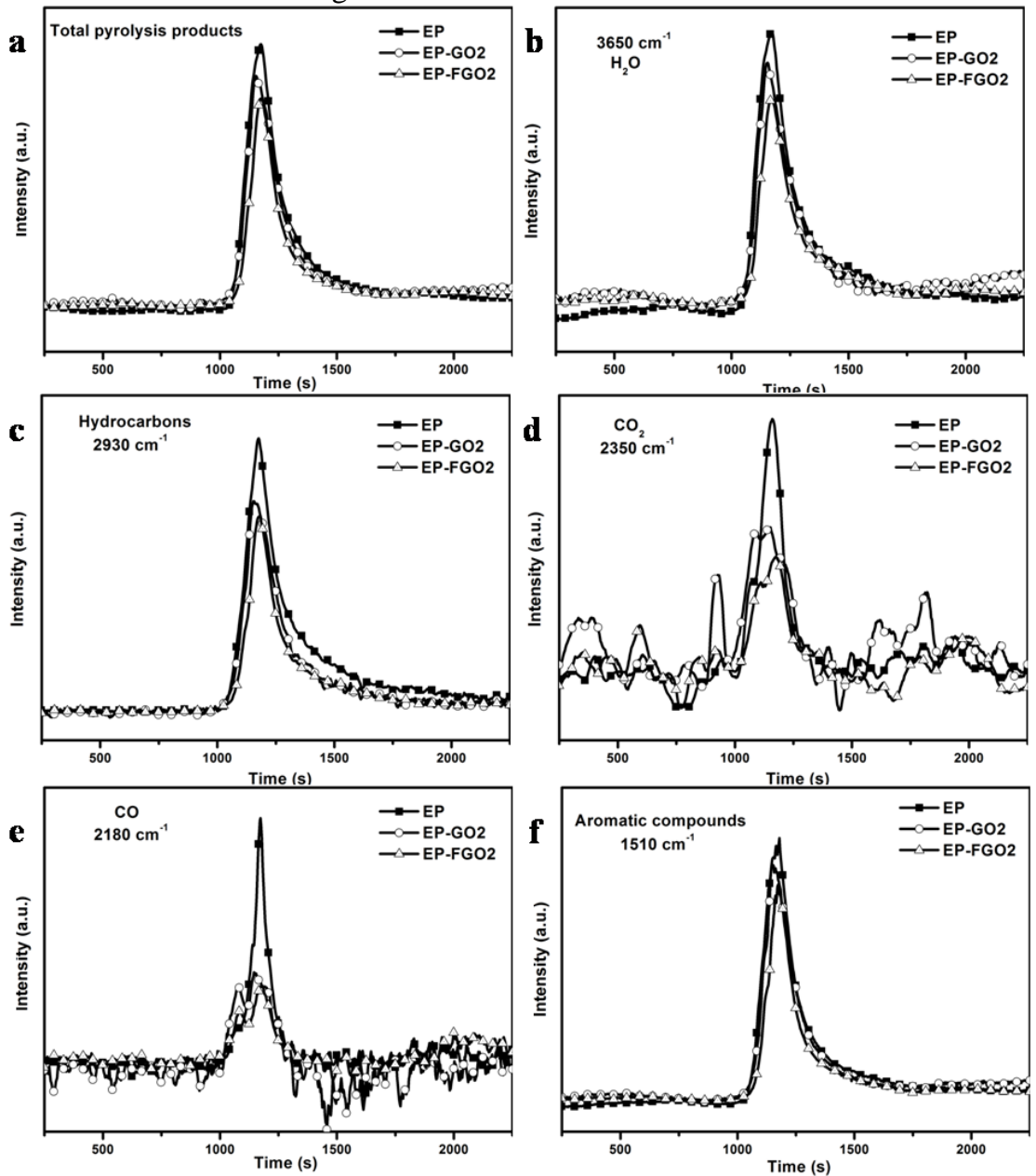

Figure 5. Absorbance of pyrolysis products for EP nanocomposites vs time: (a) total pyrolysis products; (b) $\mathrm{H}_{2} \mathrm{O}$; (c) hydrocarbons; (d) $\mathrm{CO} 2$; (e) $\mathrm{CO}$ and (d) aromatic compounds.

The gas products during the thermal degradation were analyzed by TG-FTIR measurement. As for FTIR, the characterized peaks at $3650,2930,2360,2310$ and $1510 \mathrm{~cm}^{-1}$ were attributed to $\mathrm{H}_{2} \mathrm{O}$, the hydrocarbons compounds, $\mathrm{CO}_{2}, \mathrm{CO}$, aromatic compounds of the gaseous decomposition. It was well-known that decomposition was the main process associated with the thermal degradation of polymers. In the process of decomposition of EP nanocomposites, the main decomposition products were $\mathrm{H}_{2} \mathrm{O}$, the hydrocarbons compounds, $\mathrm{CO}_{2}, \mathrm{CO}$, aromatic compounds, and etc.

The absorbance of temporal evolution of pyrolysis products for EP, EP-GO2 and EP-FGO2 were evaluated in Figure 5. It showed that the pyrolysis products for EP began to release at about $16 \mathrm{~min}$, whereas those for EP-GO2 and EP-FGO2 were decomposition early, due to the pyrolysis of functional groups on the surface of GO. However, the absorbance intensity of pyrolysis products for EP-GO2 and EP-FGO2 was lower than that for EP. It could be interpreted that the GO had the capability to prevent the pyrolysis of matrix, and the flame retardant modified GO had more effective efficiency to restrain the pyrolysis of matrix. Consequently, the GO and FGO added could reduce the release of combustible gas and the weight loss, and the results of the pyrolysis products release corresponded well to thermal analysis. 
Flame retardancy of EP-GO and EP-FGO nanocomposites
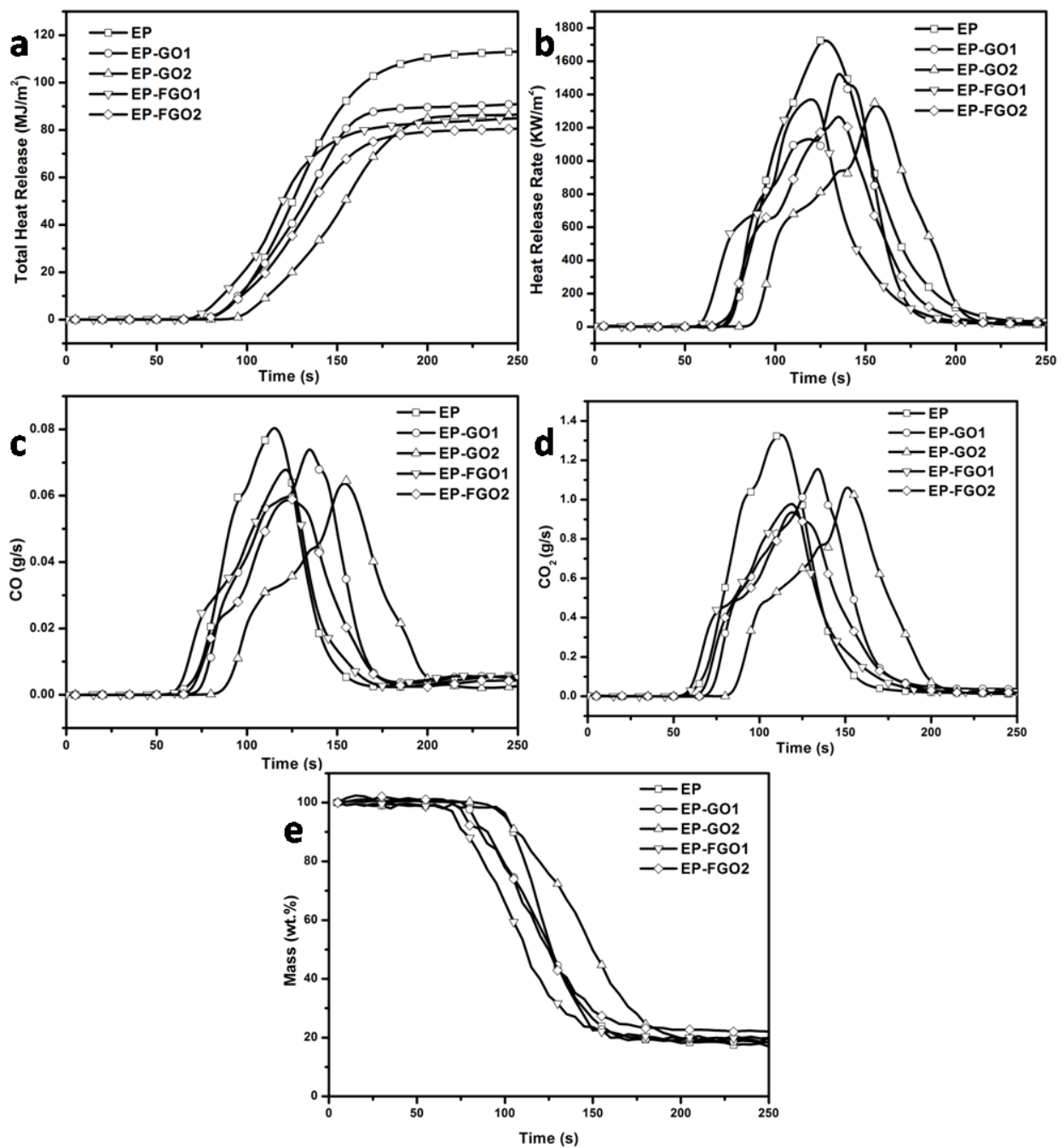

Figure 6. Cone calorimeter test results: the temporal evolution of (a) HRR curves; (b) THR curves; (c) $\mathrm{CO}_{2}$; (d) $\mathrm{CO}$ yield and (e) mass of EP nanocomposites.

Table 3 The related cone calorimeter data of EP-GO and EP-FGO nanocomposites.

\begin{tabular}{|c|c|c|c|c|c|c|c|}
\hline Sample & $\begin{array}{c}\text { Time to } \\
\text { PHRR (s) }\end{array}$ & $\begin{array}{c}\text { PHRR } \\
\left(\mathrm{KW} / \mathbf{m}^{2}\right)\end{array}$ & $\begin{array}{c}\text { THR } \\
\left(\mathbf{M J} / \mathbf{m}^{2}\right)\end{array}$ & $\begin{array}{c}\text { Average EHC } \\
(\mathrm{MJ} / \mathrm{kg})\end{array}$ & $\begin{array}{l}\text { P-CO } \\
\left(\mathrm{g} \mathrm{s}^{-1}\right)\end{array}$ & $\begin{array}{c}\mathrm{P}-\mathrm{CO}_{2}{ }^{\mathrm{a}} \\
\left(\mathrm{g} \mathrm{s}^{-1}\right)\end{array}$ & $\begin{array}{c}\mathrm{CO} / \mathrm{CO}_{2} \\
(\%)\end{array}$ \\
\hline EP & 130 & 1730 & 114.2 & 16.62 & 0.83 & 1.34 & 6.60 \\
\hline EP-GO1 & 135 & 1582 & 92.5 & 16.48 & 0.76 & 1.19 & 7.34 \\
\hline EP-GO2 & 155 & 1348 & 87.1 & 14.83 & 0.65 & 1.08 & 7.03 \\
\hline EP-FGO1 & 120 & 1406 & 86.1 & 14.12 & 0.69 & 1.03 & 7.03 \\
\hline EP-FGO2 & 135 & 1297 & 81.4 & 11.71 & 0.60 & 0.95 & 6.33 \\
\hline
\end{tabular}

${ }^{\mathrm{a}}$ The peak values of the $\mathrm{CO}$ release rate and $\mathrm{CO}_{2}$ release rate, respectively.

Cone calorimeter is a useful bench-scale tool as a test to analyze combustion behaviors and fire safety of materials. Figure 6 showed the temporal evolution of heat release rate (HRR), total heat release (THR) curves, $\mathrm{CO}_{2}$ yield, $\mathrm{CO}$ yield, mass and the related data of EP-GO and EP-FGO nanocomposites were presented in Table 3. It could be obviously seen that the THR, peak heat release rate (PHRR), average effective heat of combustion (EHC), peak values of the $\mathrm{CO}$ release rate and $\mathrm{CO}_{2}$ release rate were decreased after adding GO and FGO. It was attributed to the physical barrier effect of GO or FGO and the strong interfacial interactions between GO or FGO and EP. As compared to the neat EP, the maximum reduction was occurred in EP-FGO2, 
of which the PHRR, THR, average EHC, P- $\mathrm{CO}_{2}$, and P-CO decreased by $25 \%, 28 \%, 29.5 \%, 27 \%$ and $29 \%$, respectively. With the GO loading amount of 1 to 2 wt.\%, the PHRR and THR decreased by about $15 \%$ and $6 \%$, respectively, and the similar trend was observed for FGO-epoxy nanocomposites. FGO performed much better than GO to improve the fire safety properties of EP from the cone calorimeter data. In the case of $1 \%$ loading for the GO and FGO, the value of PHRR, THR, P-CO $\mathrm{CO}_{2}$, and P-CO was decreased by $11 \%, 7 \%, 10 \%$ and $13 \%$, respectively. The value of $\mathrm{CO} / \mathrm{CO}_{2}$ ratios showed that $\mathrm{EP}-\mathrm{FGO} 2$ displayed the best extent of combustion than other samples. It may be due to that the flame retardant grafted on FGO catalyzed the more char formation of EP-FGO nanocomposites than that of GO, and then the char as the barrier restrained the release of $\mathrm{CO}$ and $\mathrm{CO}_{2}$. The time to ignition (TTI) was delayed when the loading amount increased, however, the TTI value was decreased for FGO. It was attributed to the pyrolysis of the flame retardant grafted on the surface. The mass loss curve showed that the char residual of EP-FGO was more than EP and EP-GO and the time of decomposition was also delayed compared to EP and EP-GO, suggesting the results of TGA measurement. The results indicated that flame retardant modified GO lead to decrease of THR of nanocomposites because of more char residue produced to isolate the heat release. Therefore, the addition of GO enhanced the fire safety properties of the composites, and the EP-FGO displayed the better fire safety properties.

\section{CONCLUSION}

In this paper, functionalized graphene oxide with flame retardant was synthesized and then applied to prepare the epoxy nanocomposites via in situ polymerization. The results of FTIR, ${ }^{1} \mathrm{H}$ NMR, ${ }^{31} \mathrm{P}$ NMR and XPS indicated that the PDBMP was successful synthesized and covalently grafted onto the surface of GO. The TGA and TG-IR data showed that thermal stability of graphene oxide was improved after the functionalization. The incorporation of FGO into EP obviously improved the char yields and the thermal stability compared with EP-GO nanocomposites. The char formed as the physical barrier effect of GO or FGO had a great effect of flame retardancy of EP nanocomposites, of which the PHRR, THR, EHC, $\mathrm{CO}_{2}$ yield and $\mathrm{CO}$ yield were decreased after adding GO and FGO. In conclusion, this work represents that functionalized GO with flame retardant is an effective additive to enhance the thermal stability and fire safety properties of polymers.

\section{ACKNOWLEDGEMENTS}

The work was financially supported the joint fund of NSFC and Guangdong Province (No. U1074001), State Key Laboratory of Environmental Adaptability for Industrial Products, China National Electric Apparatus Research Institute, Guangzhou, 510300, P.R. China, the youth innovation fund of USTC and the Program for Science and Technology of SuZhou (SG-0841), Opening Project of State Key Laboratory of Fire Science of USTC (No. HZ2011-KF05).

\section{REFERENCES}

[1] Xiao H., et al., (2012) Graphene-based composites, Chemical Society Reviews, 41: 666-686, http://pubs.rsc.org/en/Content/ArticleLanding/2012/CS/c1cs15078b.

[2] Hyunwoo K., et al., (2010) Graphene/Polymer Nanocomposites, Macromolecules, 43:6515-6530, http://pubs.acs.org/doi/abs/10.1021/ma100572e.

[3] Kian P., et al., (2010) The chemistry of graphene, Journal of Materials Chemistry, 20: 22772289, http://pubs.rsc.org/en/Content/ArticleLanding/2010/JM/b920539j.

[4] Ming F., et al., (2009) Covalent polymer functionalization of graphene nanosheets and mechanical properties of composites, Journal of Materials Chemistry, 19: 7098-7105, http://pubs.rsc.org/en/content/articlehtml/2009/jm/b908220d.

[5] Chao W., et al., (2012) Hyperbranched-polymer functionalization of graphene sheets for enhanced mechanical and dielectric properties of polyurethane composites, Journal of Materials Chemistry, 22: 7010-7019, http://pubs.rsc.org/en/content/articlehtml/2012/jm/c2jm16901k.

[6] Horacio J. S., et al., (2009) Polymeric Modification of Graphene through Esterification of Graphite Oxide and Poly(vinyl alcohol), Macromolecules, 42: 6331-6334, http://pubs.acs.org/doi/abs/10.1021/ma900845w.

[7] Hyunwoo K., et al., (2010) Graphene/Polyurethane Nanocomposites for Improved Gas Barrier and Electrical Conductivity, Chemistry of $\quad$ Materials, 22:3441-3450, http://pubs.acs.org/doi/abs/10.1021/cm100477v.

[8] Guobo H., et al., (2012) How can graphene reduce the flammability of polymer nanocomposites, Materials Letters, 66, 187-189, http://www.sciencedirect.com/science/article/pii/S0167577X11009669. 
[9] Xin W., et al., (2013) Self-assembly of Ni-Fe layered double hydroxide/graphene hybrids for reducing fire hazard in epoxy composites, Journal of Materials Chemistry A, 1: 4383-4390, http://pubs.rsc.org/en/content/ articlehtml/2013/ta/c3ta00035d.

[10] Xin W., et al., (2012) Simultaneous reduction and surface functionalization of graphene oxide with POSS for reducing fire hazards in epoxy composites, Journal of Materials Chemistry, 22: 22037-22043, http:// pubs.rsc.org/en/content/articlehtml/2012/jm/c2jm35479a.

[11] Chenlu B., et al., (2012) Functionalized graphene oxide for fire safety applications of polymers: a combination of condensed phase flame retardant strategies, Journal of Materials Chemistry, 22: 23057-23063, http://pubs.rsc.org/en/content/articlehtml/2012/jm/c2jm35001g.

[12] Guobo H., et al., (2012) A novel intumescent flame retardant-functionalized graphene: Nanocomposite synthesis, characterization, and flammability properties, Materials Chemistry and Physics, 135, 938-947, http:// www.sciencedirect.com/science/article/pii/S0254058412005548.

[13] Jun Z., et al., (2008) Graphene production: New solutions to a new problem, Nature Nanotechnology, 3, 528-529, http://www.nature.com/nnano/journal/v3/n9/full/nnano.2008.249.html.

[14] Ramanathanf T., et al., (2008) Functionalized graphene sheets for polymer nanocomposites, Nature Nanotechnology, 3: 327-331, http://www.nature.com/nnano/journal/v3/n6/abs/nnano.2008.96.html.

[15] Sandip N., et al., (2006) Solution Properties of Graphite and Graphene, Journal of the American Chemical Society, 128: 7720-7721, http://pubs.acs.org/doi/abs/10.1021/ja060680r.

[16] Wenchao Z., et al., (2011) Pyrolysis and fire behaviour of epoxy resin composites based on a phosphoruscontaining polyhedral oligomeric silsesquioxane (DOPO-POSS), Polymer Degradation and Stability, 96:

1821-1832, http://www.sciencedirect.com/science/article/pii/S0141391011002424.

[17] Chin L. C., et al., (2008) Synthesis, characterization and properties of novel self-extinguishing organic-inorganic nanocomposites containing nitrogen, silicon and phosphorus via sol-gel method, $\begin{array}{lllll}\text { Composites Science } & \text { and }\end{array}$ http://www.sciencedirect.com/science/article/pii/S0014305702001234.

[18] Pingan S., et al., (2009) Effects of Reactive Compatibilization on the Morphological, Thermal, Mechanical, and Rheological Properties of Intumescent Flame-Retardant Polypropylene, ACS Applied Materials Interfaces, 2: 452-459, http://pubs.acs.org/doi/abs/10.1021/am8001204.

[19] Jose Alcon M., et al., (2011) Synthesis, Characterization and Polymerization of a Novel Glycidyl Phosphinate, Macromolecular Rapid Communication, 22:1265-1271, http://onlinelibrary.wiley.com/doi/10.1002/1521-3927(20011001)22:15\%3C1265::AID-MARC1265\%3E3.0.C O;2-K/abstract.

[20] Chun S. W., et al., (2000) Novel phosphorus-containing epoxy resins.Part II: curing kinetics, Polymer, 41: 8579-8586, http://www.sciencedirect.com/science/article/pii/S0032386100002111.

[21] Tsung H. H., et al., (2009) Thermal, physical and flame-retardant properties of phosphorus-containing epoxy cured with cyanate ester, Reactive and Functional Polymers, 69: 176-182, http://www.sciencedirect.com/ science/article/pii/S1381514808002186.

[22] Ying L. L., et al., (2002) Flame-Retardant Epoxy Resins from o-Cresol Novolac Epoxy Cured witha Phosphorus-Containing Aralkyl Novolac, Journal of Polymer Science: Part A: Polymer Chemistry, 40: 2329-2339, http://onlinelibrary.wiley.com/doi/10.1002/pola.10320/full.

[23] Chun S. W., et al., (1999) Properties and Curing Kinetic of Diglycidyl Ether of Bisphenol A Cured with a Phosphorus-Containing Diamine, Journal of Applied Polymer Science, 74: 1635-1645, http://onlinelibrary.wiley.com/doi/10.1002/(SICI)1097-4628(19991114)74:7\%3C1635::AID-APP4\%

3E3.0.CO ;2-P/abstract.

[24] Jeng Y. S., et al., (2001) Synthesis of novel flame retardant epoxy hardeners and properties of cures products, Polymer, 42: 7617-7625, http://www.sciencedirect.com/science/article/pii/S0032386101002579.

[25] Dechao S., et al., (2011) Synthesis of three novel phosphorus-containing flame retardants and their application in epoxy resins, Polymer Degradation and Stability, 96: 1720-1724, http://www.sciencedirect.com/ science/article/pii/S0141391011002734.

[26] Li P. G., et al., (2008) A flame-retardant epoxy resin based on a reactive phosphorus-containing monomer of DODPP and its thermal and flame-retardant properties, Polymer Degradation and Stability, 93: 1308-1315, http://www.sciencedirect.com/science/article/pii/S0141391008001092.

[27] Schartel B., et al., (2008) Pyrolysis and fire behaviour of epoxy systems containing a novel 9,10dihydro-9-oxa-10-phosphaphenanthrene-10-oxide-(DOPO)-based diamino hardener, European Polymer Journal,44: 704-715, http://www.sciencedirect.com/science/article/pii/S0014305708000256. 
[28] Mercado L.A., et al., (2006) Silicon-containing flame retardant epoxy resins: Synthesis, characterization and properties, Polymer Degradation and Stability, 91: 2588-2594, http://www.sciencedirect.com/science/ article/pii/S0141391006001765.

[29] Hummers,W. S., et al., (1958) Preparation of graphitic oxide, Journal of American Chemistry Society, 80: 1339, http://pubs.acs.org/doi/abs/10.1021/ja01539a017.

[30] Xin W., et al., (2010) Flame retardancy and thermal degradation mechanism of epoxy resin composites based on a DOPO substituted organophosphorus oligomer, Polymer, 51: 2435-2445, http://www.sciencedirect.com/science/article/pii/S0032386110002806.

[31] Sasha S., et al., (2006) Synthesis and exfoliation of isocyanate-treated graphene oxide nanoplatelets, Carbon, 44: 3342-3347, http://www.sciencedirect.com/science/article/pii/S0008622306003307.

[32] Tang X.Z., et al., (2011) Enhanced thermal stability in graphene oxide covalently functionalized with 2-amino-4,6-didodecylamino-1,3,5-triazine, Carbon, 49: $1258-1265$, http://www.sciencedirect.com/science/article/pii/S0008622310008535.

[33] Yang H.F., et al., (2009) Covalent functionalization of chemically converted graphene sheets via silane and its reinforcement, Journal of Materials chemistry, 19: 4632-4638, http://pubs.rsc.org/en/Content/ArticleLanding/2009/JM/B901421G\#!divAbstract. 